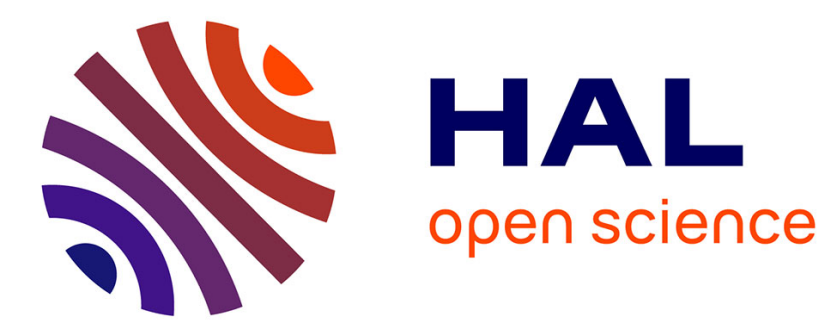

\title{
Deterministic Equivalents for the Performance Analysis of Isometric Random Precoded Systems
}

Jakob Hoydis, Romain Couillet, Merouane Debbah

\section{To cite this version:}

Jakob Hoydis, Romain Couillet, Merouane Debbah. Deterministic Equivalents for the Performance Analysis of Isometric Random Precoded Systems. IEEE International Conference on Communications 2011, Jun 2011, Kyoto, Japan. 5 p., 10.1109/icc.2011.5962503 . hal-00648021

\section{HAL Id: hal-00648021}

https://hal-centralesupelec.archives-ouvertes.fr/hal-00648021

Submitted on 4 Dec 2011

HAL is a multi-disciplinary open access archive for the deposit and dissemination of scientific research documents, whether they are published or not. The documents may come from teaching and research institutions in France or abroad, or from public or private research centers.
L'archive ouverte pluridisciplinaire $\mathbf{H A L}$, est destinée au dépôt et à la diffusion de documents scientifiques de niveau recherche, publiés ou non, émanant des établissements d'enseignement et de recherche français ou étrangers, des laboratoires publics ou privés. 


\title{
Deterministic Equivalents for the Performance Analysis of Isometric Random Precoded Systems
}

\author{
Jakob Hoydis ${ }^{* \dagger}$, Romain Couillet ${ }^{\ddagger}$, and Mérouane Debbah ${ }^{\dagger}$ \\ *Department of Telecommunications, Supélec, Gif-sur-Yvette, France \\ ${ }^{\dagger}$ Alcatel-Lucent Chair on Flexible Radio, Supélec, Gif-sur-Yvette, France \\ $\ddagger$ EDF Chair on System Sciences and the Energy Challenge, Centrale Paris-Supélec, France \\ \{jakob.hoydis, romain.couillet, merouane.debbah\}@ supelec.fr
}

\begin{abstract}
We consider a general wireless channel model for different types of code-division multiple access (CDMA) and space-division multiple-access (SDMA) systems with isometric random signature/precoding matrices over frequency-selective and flat fading channels. We derive deterministic approximations of the Stieltjes transform, the mutual information and the signal-to-interference-plus-noise ratio (SINR) at the output of the minimum-mean-square-error (MMSE) receiver and provide a simple fixed-point algorithm for their computation, which is proved to converge. The deterministic approximations are asymptotically tight, almost surely, but shown by simulations to be very accurate for even small system dimensions. Our analysis requires neither arguments from free probability theory nor the asymptotic freeness or the convergence of the spectral distribution of the involved matrices. The results presented in this work are, therefore, also a novel contribution to the field of random matrix theory and might be useful to further applications involving isometric random matrices.
\end{abstract}

\section{INTRODUCTION}

Consider the following time-discrete wireless channel

$$
\mathbf{y}=\sum_{k=1}^{K} \mathbf{H}_{k} \mathbf{W}_{k} \mathbf{P}_{k}^{\frac{1}{2}} \mathbf{x}_{k}+\mathbf{n}
$$

where

(i) $\mathbf{y} \in \mathbb{C}^{N}$ is the channel output vector.

(ii) $\mathbf{H}_{k} \in \mathbb{C}^{N \times N}, k=1, \ldots, K$, are non-random complex channel matrices.

(iii) $\mathbf{W}_{k} \in \mathbb{C}^{N \times n_{k}}, k=1, \ldots, K$, are complex signature/precoding matrices which contain each $n_{k} \leq N$ orthonormal columns of independent $N \times N$ Haardistributed random unitary matrices.

(iv) $\mathbf{P}_{k} \in \mathbb{R}_{+}^{n_{k} \times n_{k}}, k=1, \ldots, K$, are non-random nonnegative diagonal matrices.

(v) $\mathbf{x}_{k} \in \mathbb{C}^{n_{k}}, k=1, \ldots, K$, are random transmit vectors, having independent and identically distributed (i.i.d.) elements with zero mean and unit variance.

(vi) $\mathbf{n} \in \mathbb{C}^{N}$ is a noise vector having i.i.d. circular-symmetric complex Gaussian entries with zero mean and variance $\rho$.

Possible applications of this channel model arise in the study of direct-sequence (DS) or multi-carrier (MC) code-division multiple-access (CDMA) systems with isometric signatures over frequency-selective fading channels or space-division multiple-access (SDMA) systems with isometric precoding matrices over flat-fading channels. More precisely, for DSCDMA systems, the $\mathbf{H}_{k}$ are either Toeplitz or circular matrices (if a cyclic prefix is used) constructed from the channel impulse response; for MC-CDMA, the matrices $\mathbf{H}_{k}$ are diagonal and represent the channel frequency response on each subcarrier; for flat fading SDMA systems, the matrices $\mathbf{H}_{k}$ can be of arbitrary form and their elements represent the complex channel gains between the transmit and receive antennas. In all cases, the diagonal entries of the matrices $\mathbf{P}_{k}$ determine the transmit power of each signature (CDMA) or transmit stream (SDMA). Specific scenarios to which the channel model applies are:

- single/multi-cell uplink DS/MC-CDMA with multiple transmit signatures per user

- single/multi-cell downlink DS/MC-CDMA with single/multiple transmit signatures per user

- single/multi-cell uplink SDMA with unitary precoding codebooks and multiple streams per user

- single/multi-cell downlink SDMA with unitary precoding codebooks and single/multiple streams per user.

The large system analysis of random i.i.d. and random orthogonal precoded systems with optimal and sub-optimal linear receivers has been the subject of numerous publications. The asymptotic performance of minimum-mean-square-error (MMSE) receivers for the channel model (1) for the case $K=1, \mathbf{P}=\mathbf{I}_{n_{1}}$ and $\mathbf{H}$ diagonal with i.i.d. elements has been studied in [1] relying on results from free probability theory. This result was extended to frequency-selective fading channels and sub-optimal receivers in [2]. The case of i.i.d. and isometric MC-CDMA over Rayleigh fading channels with multiple signatures per user terminal, i.e., $K \geq 1$ and $\mathbf{H}_{k}$ diagonal with i.i.d. complex Gaussian entries, was considered in [3], where approximate solutions of the signalto-noise-plus-interference-ratio (SINR) at the output of the MMSE receiver were provided. Asymptotic expressions for the spectral efficiency of the same model were then derived in [4]. DS-CDMA over flat-fading channels, i.e., $K \geq 1$, $n_{k}=N$ and $\mathbf{H}_{k}=\mathbf{I}_{N}$ for all $k^{*}$, was studied in [5], where the authors derive deterministic equivalents of the Shannonand $\eta$-transform based on the asymptotic freeness [6] of the matrices $\mathbf{W}_{k} \mathbf{P}_{k} \mathbf{W}_{k}^{\mathrm{H}}$. Moreover, a sum-rate maximizing power-allocation algorithm was proposed. Finally, a different 
approach via incremental matrix expansion [7] led to the exact characterization of the asymptotic SINR of the MMSE receiver for the general channel model (1). However, the previously mentioned works share the underlying assumption that the spectral distributions of the matrices $\mathbf{H}_{k}$ and $\mathbf{P}_{k}$ converge to some limiting distributions and/or the matrices $\mathbf{H}_{k} \mathbf{H}_{k}^{\mathrm{H}}$ are jointly diagonalizable. ${ }^{1}$ Moreover, the computation of the asymptotic SINR requires the computation of rather complicated implicit equations. These can be solved in most cases by standard fixed-point algorithms but a proof of convergence to the correct solution has not been provided yet. Moreover, a closed-form expression for the asymptotic spectral efficiency is missing, although an approximate solution which requires a numerical integration was presented in [4].

Recently, unitary precoders gained also significant interest for spatial multiplexing systems [8] and are now proposed as limited feedback beamforming solutions in future wireless standards [9]. Thus, the performance evaluation of such systems is compulsory and a field of active research [10]. However, little related analytical work based on large random matrix theory has been published so far and the results presented in this paper might stimulate further research in this direction.

Before we summarize the main results of this work, we need the following definitions. Let $\mathbf{B}$ be the $N \times N$ complex matrix

$$
\mathbf{B}=\sum_{k=1}^{K} \mathbf{H}_{k} \mathbf{W}_{k} \mathbf{P}_{k} \mathbf{W}_{k}^{\mathrm{H}} \mathbf{H}_{k}^{\mathrm{H}}
$$

and, for $z \in \mathbb{C} \backslash \mathbb{R}_{+}$, denote by $m(z)$ the Stieltjes transform [6] of the empirical spectral distribution (e.s.d.) $F$ of $\mathbf{B}$, given as

$$
m(z)=\frac{1}{N} \operatorname{tr}\left(\mathbf{B}-z \mathbf{I}_{N}\right)^{-1}=\int \frac{1}{\lambda-z} d F(\lambda)
$$

Moreover, $I(\rho)$ denotes the normalized mutual information ${ }^{2}$ of the channel (1) assuming complex Gaussian input vectors $\mathbf{x}_{k}$, given by [11]

$$
I(\rho)=\frac{1}{N} \log \operatorname{det}\left(\mathbf{I}_{N}+\frac{1}{\rho} \sum_{k=1}^{K} \mathbf{H}_{k} \mathbf{W}_{k} \mathbf{P}_{k} \mathbf{W}_{k}^{\mathrm{H}} \mathbf{H}_{k}^{\mathrm{H}}\right)
$$

expressed in nats/s. We further denote by $\gamma_{k j}$ the SINR at the output of the linear MMSE receiver for the $j$ th component of transmit vector $\mathbf{x}_{k}$, which reads [12]

$$
\gamma_{k j}=p_{k j} \mathbf{w}_{k j}^{\mathrm{H}} \mathbf{H}_{k}^{\mathrm{H}}\left(\mathbf{B}_{[k j]}+\rho \mathbf{I}_{N}\right)^{-1} \mathbf{H}_{k} \mathbf{w}_{k j}
$$

where $\mathbf{B}_{[k j]}=\mathbf{B}-p_{k j} \mathbf{H}_{k} \mathbf{w}_{k j} \mathbf{w}_{k j}^{\mathrm{H}} \mathbf{H}_{k}^{\mathrm{H}}, p_{k j}$ is the $j$ th diagonal entry of $\mathbf{P}_{k}$ and $\mathbf{w}_{k j}$ is the $j$ th column of $\mathbf{W}_{k}$.

The contribution of this paper is twofold. As a contribution to the field of random matrix theory, we provide a deterministic equivalent $\bar{m}(z)$ to $m(z)$, such that, when $N$ and all $n_{k}$ grow

\footnotetext{
${ }^{1}$ That is, there exists a unitary matrix $\mathbf{V}$ such that $\mathbf{V} \mathbf{H}_{k} \mathbf{H}_{k}^{\mathrm{H}} \mathbf{V}^{\mathrm{H}}$ is diagonal for all $k$.

${ }^{2}$ This expression corresponds to the mutual information per chip for CDMA systems and to the mutual information per receive-antenna in SDMA systems.
}

large $m(z)-\bar{m}(z) \stackrel{\text { a.s. }}{\longrightarrow} 0 .{ }^{3}$ Denote $\bar{F}$ the distribution function with Stieltjes transform $\bar{m}(z)$. The previous result establishes also that, asymptotically, $F-\bar{F} \Rightarrow 0$, almost surely. Although deterministic equivalents of Stieltjes transforms are by now more or less standard and have been developed for rather involved random matrix models [13], [14], results for the case of Haar distributed matrices are still an exception. In particular, most results on Haar matrices are based on the assumption of asymptotic freeness (see [6, Chapter 3]) of the concerned matrices, a requirement which is rarely met for the matrices of our model. The approach taken in this work is, thus, novel as it does not rely on free probability theory and we do not require any of the matrices in (1) to be asymptotically free.

As a contribution to the field of wireless communications, we derive deterministic approximations $\bar{I}(\rho)$ and $\bar{\gamma}_{k j}$ of $I(\rho)$ and $\gamma_{j k}$, respectively, which are asymptotically accurate, almost surely. In contrast to existing works, (i) our deterministic equivalents are easy to compute as we provide a simple fixed-point algorithm which is proved to converge, (ii) the deterministic approximation $\bar{I}(\rho)$ of $I(\rho)$ is given in closed form and does not require any numerical integration, (iii) we do not require that the spectral distributions of the matrices $\mathbf{H}_{k}$ and $\mathbf{P}_{k}$ converge or that the matrices $\mathbf{H}_{k} \mathbf{H}_{k}^{\mathrm{H}}$ are jointly diagonalizable.

\section{MAIN RESUlTS}

In this section, we present the main results of the paper. We first need to introduce some technical conditions and definitions:

The notation $N \rightarrow \infty$ will denote in the sequel that $N$ and $n_{k}, 1 \leq k \leq K$, grow large with ratios $c_{k}=\frac{n_{k}}{N}$, such that $0 \leq c_{k} \leq 1$, for all $N, n_{k}$. Denote by $\left\|\mathbf{H}_{k}\right\|$ the spectral norm of the matrix $\mathbf{H}_{k}$. We assume that for all $N, n_{k}$, there exist non negative real numbers $h_{\max }$ and $p_{\max }$, such that

$$
\sup _{k}\left\|\mathbf{H}_{k}\right\| \leq h_{\max }, \quad \sup _{k}\left\|\mathbf{P}_{k}\right\| \leq p_{\max } .
$$

Denote by $\mathbb{C}_{+}=\{z \in \mathbb{C}: \operatorname{Im}(z)>0\}$, and by $\mathcal{S}$ the class of functions $f$ analytic over $\mathbb{C} \backslash \mathbb{R}_{+}$, such that for $z \in \mathbb{C}_{+}$, $f(z) \in \mathbb{C}_{+}$and $z f(z) \in \mathbb{C}_{+}$, and $\lim _{y \rightarrow \infty}-\mathbf{i} y f(\mathbf{i} y)=1$, where $\mathbf{i}=\sqrt{-1}$. Such functions are known to be Stieltjes transforms [6] of probability measures over $\mathbb{R}_{+}$. We are now in position to state our main results:

Theorem 1 (Fundamental equations): Assume that the conditions in (4) hold and define the matrices $\mathbf{R}_{k}=\mathbf{H}_{k} \mathbf{H}_{k}^{\mathrm{H}}, 1 \leq$ $k \leq K$. Then, for $z \in \mathbb{C} \backslash \mathbb{R}_{+}$, the following system of $K$ implicit equations in $\bar{e}_{k}(z), 1 \leq k \leq K$,

$$
\begin{aligned}
& \bar{e}_{k}(z)=\frac{1}{N} \operatorname{tr} \mathbf{P}_{k}\left(e_{k}(z) \mathbf{P}_{k}+\left[1-e_{k}(z) \bar{e}_{k}(z)\right] \mathbf{I}_{n_{k}}\right)^{-1} \\
& e_{k}(z)=\frac{1}{N} \operatorname{tr} \mathbf{R}_{k}\left(\sum_{j=1}^{K} \bar{e}_{j}(z) \mathbf{R}_{j}-z \mathbf{I}_{N}\right)^{-1}
\end{aligned}
$$

${ }^{3}$ We use $\stackrel{\text { a.s. }}{\longrightarrow}$ and $\Rightarrow$ to denote almost sure convergence and convergence in distribution, respectively. 
has a unique solution $\left(\bar{e}_{1}(z), \ldots, \bar{e}_{K}(z)\right) \in \mathcal{S}^{K}$.

Moreover, for $z<0$, the $e_{k}(z)$ and $\bar{e}_{k}(z)$ can be easily computed by the fixed-point Algorithm 1.

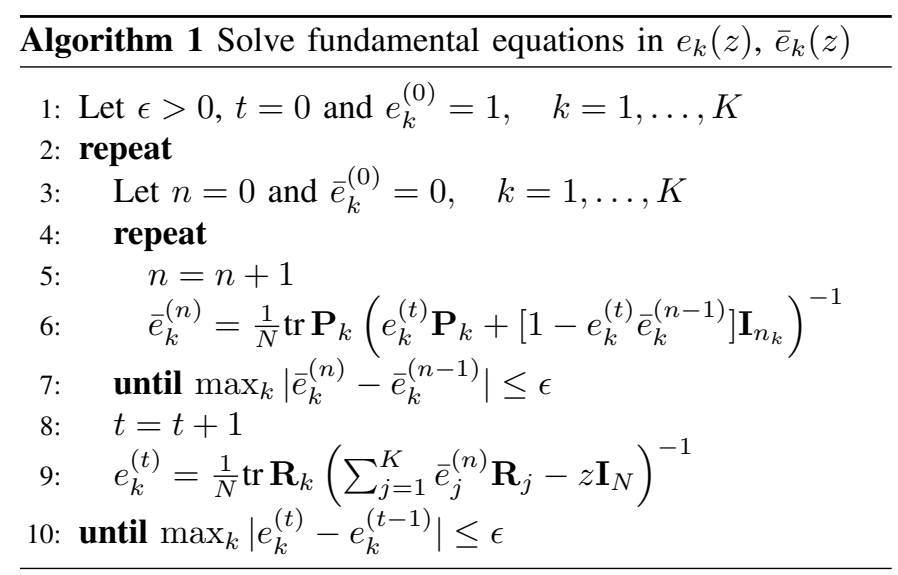

Proof: The proof is postponed to the appendix.

Theorem 2 (Deterministic equivalents): Assume that the conditions in (4) hold, define the matrices $\mathbf{R}_{k}=\mathbf{H}_{k} \mathbf{H}_{k}^{\mathrm{H}}, 1 \leq$ $k \leq K$, and let $c_{k}=\frac{n_{k}}{N}, 1 \leq k \leq K$.

(i) Let

$$
\bar{m}(z)=\frac{1}{N} \operatorname{tr}\left(\sum_{k=1}^{K} \bar{e}_{k}(z) \mathbf{R}_{k}-z \mathbf{I}_{N}\right)^{-1}
$$

where the $\bar{e}_{k}(z)$ are given by Theorem 1 . Then, for $z<0$, the following holds true

$$
m(z)-\bar{m}(z) \underset{N \rightarrow \infty}{\stackrel{\text { a.s. }}{\longrightarrow}} 0
$$

Moreover,

$$
F-\bar{F} \Rightarrow 0
$$

almost surely.

(ii) Let $\rho>0$ and denote $e_{k}=e_{k}(-\rho)$ and $\bar{e}_{k}=\bar{e}_{k}(-\rho)$. Consider the quantity:

$$
\begin{aligned}
\bar{I}(\rho)=\frac{1}{N} & \log \operatorname{det}\left(\mathbf{I}_{N}+\frac{1}{\rho} \sum_{k=1}^{K} \bar{e}_{k} \mathbf{R}_{k}\right) \\
& +\frac{1}{N} \sum_{k=1}^{K} \log \operatorname{det}\left(\left[1-e_{k} \bar{e}_{k}\right] \mathbf{I}_{n_{k}}+e_{k} \mathbf{P}_{k}\right) \\
& +\sum_{k=1}^{K}\left(1-c_{k}\right) \log \left(1-e_{k} \bar{e}_{k}\right)
\end{aligned}
$$

where the $e_{k}$ and $\bar{e}_{k}$ are given by Theorem 1 . Then, the following holds true

$$
I(\rho)-\bar{I}(\rho) \underset{N \rightarrow \infty}{\stackrel{\text { a.s. }}{\longrightarrow}} 0 .
$$

(iii) Let $\rho>0$, and define $e_{k}=e_{k}(-\rho)$ and $\bar{e}_{k}=\bar{e}_{k}(-\rho)$, given by Theorem 1. Further denote

$$
\bar{\gamma}_{k j}=p_{k j} \frac{e_{k}}{1-\bar{e}_{k} e_{k}} \text {. }
$$

Then,

$$
\gamma_{k j}-\bar{\gamma}_{k j} \underset{N \rightarrow \infty}{\stackrel{\text { a.s. }}{\longrightarrow}} 0
$$

Proof: The proof is postponed to the appendix.

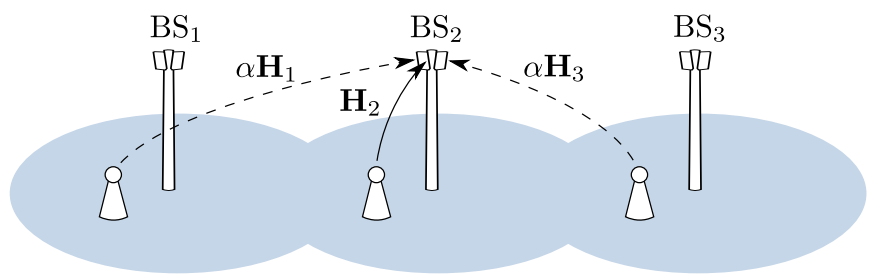

Fig. 1: Three cell example: $\mathrm{BS}_{2}$ decodes the $n$ streams from the UT in its own cell while treating the other signals as interference.

\section{NUMERICAL RESULTS}

We will now demonstrate the accuracy of the deterministic approximations by providing some simulation results. Consider the three-cell uplink channel from $K=3$ user terminals (UTs) to three base stations (BSs) as shown in Fig. 1. We focus on the center cell $\mathrm{BS}_{2}$ and assume that the BSs only decode the signals received from the UT in their own cell. The received signal at $\mathrm{BS}_{2}$ reads

$$
\mathbf{y}=\mathbf{H}_{2} \mathbf{W}_{2} \mathbf{x}_{2}+\underbrace{\alpha \mathbf{H}_{1} \mathbf{W}_{1} \mathbf{x}_{1}+\alpha \mathbf{H}_{3} \mathbf{W}_{3} \mathbf{x}_{3}+\mathbf{n}}_{\triangleq \mathbf{z}}
$$

where $0 \leq \alpha \leq 1$ is an inter-cell interference factor and the vector $\mathbf{z} \in \mathbb{C}^{\bar{N}}$ combines the inter-cell interference and the thermal noise. The covariance matrix $\mathbf{Z} \in \mathbb{R}_{+}^{N \times N}$ of $\mathbf{z}$ is given as

$$
\mathbf{Z}=\mathbb{E}\left[\mathbf{z z}^{\mathrm{H}}\right]=\alpha^{2} \sum_{i=1, i \neq 2}^{3} \mathbf{H}_{i} \mathbf{W}_{i} \mathbf{P}_{i} \mathbf{W}_{i}^{\mathrm{H}} \mathbf{H}_{i}^{\mathrm{H}}+\rho \mathbf{I}_{N} .
$$

We assume a DS-CDMA system with cyclic prefix so that the channel matrices $\mathbf{H}_{k} \in \mathbb{C}^{N \times N}$ have a circular structure as given by

$$
\mathbf{H}_{k}=\left(\begin{array}{ccccccc}
h_{k, 1} & 0 & \cdots & 0 & h_{k, L} & \cdots & h_{k, 2} \\
\vdots & \ddots & \ddots & & \ddots & \ddots & \vdots \\
h_{k, L} & & \ddots & \ddots & & \ddots & h_{k, L} \\
0 & \ddots & & \ddots & \ddots & & 0 \\
\vdots & \ddots & \ddots & & \ddots & \ddots & \vdots \\
\vdots & & \ddots & \ddots & & \ddots & 0 \\
0 & \cdots & \cdots & 0 & h_{k, L} & \cdots & h_{k, 1}
\end{array}\right)
$$

where $L \leq N$ is the delay spread and the channel taps $h_{k, j} \sim \mathcal{C N}(0,1)$ are i.i.d. over $k, j$. For simplicity, we further assume that each UT uses $n_{k}=n$ different transmit signatures to which it assigns equal power, i.e., $\mathbf{P}_{k}=\frac{P}{n} \mathbf{I}_{n}$. Assuming Gaussian signaling, the achievable sum-rate of the center cell $I(\rho)$ is given by

$$
\begin{aligned}
I(\rho) & =\frac{1}{N} \log \operatorname{det}\left(\mathbf{I}_{N}+\frac{P}{n} \mathbf{Z}^{-\frac{1}{2}} \mathbf{H}_{2} \mathbf{W}_{2} \mathbf{W}_{2}^{\mathrm{H}} \mathbf{H}_{2} \mathbf{Z}^{-\frac{1}{2}}\right) \\
& =\frac{1}{N} \log \operatorname{det}\left(\frac{\mathbf{Z}}{\rho}+\frac{P}{\rho n} \mathbf{H}_{2} \mathbf{W}_{2} \mathbf{W}_{2}^{\mathrm{H}} \mathbf{H}_{2}\right)-\frac{1}{N} \log \operatorname{det} \frac{\mathbf{Z}}{\rho} .
\end{aligned}
$$

${ }^{4}$ We have chosen this particular structure for comparison purposes with [7]. Note that the matrices $\mathbf{H}_{k} \mathbf{H}_{k}^{\mathrm{H}}$ are jointly diagonalizable by a Fourier matrix. In principle, our result holds for any other choice of $\mathbf{H}_{k}$. 


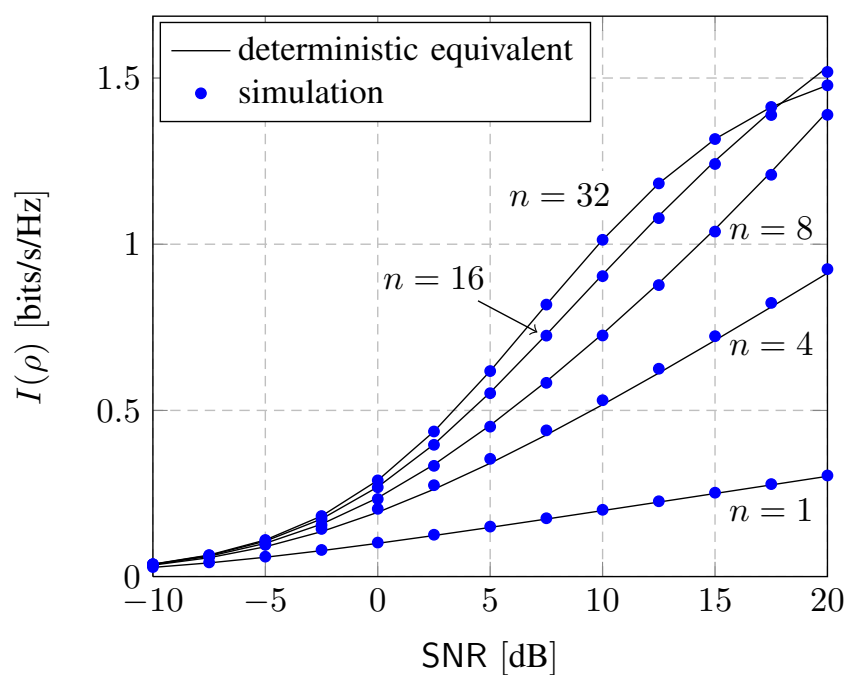

Fig. 2: Mutual Information $I(\rho)$ versus SNR for $N=32$ and different numbers of transmit signatures $n$.

Since both terms in the difference above are of the form (2), we can apply Theorem 2 (ii) to each term separately to compute a deterministic equivalent approximation of $I(\rho)$. An approximation of the SINR at the output of the MMSE receiver for the $j$ th entry of $\mathbf{x}_{2}$ (as given by (3)) can be computed directly by Theorem 2 (iii). In the sequel, we assume $P=1$, $\alpha=0.5, N=32, L=8$ and define $\mathrm{SNR}=1 / \rho$. We consider a single random realization of the matrices $\mathbf{H}_{k}$ and $\mathbf{W}_{k}$.

Fig. 2 depicts $I(\rho)$ and the deterministic equivalent $\bar{I}(\rho)$ versus SNR for different values of $n=\{1,4,8,16,32\}$. We observe a very good fit between both results over the full range of SNR and $n$. This validates the deterministic approximation for systems of even small dimensions.

In Fig. 3, we compare the SINR $\gamma_{21}$ against its deterministic approximation $\bar{\gamma}_{21}$ as a function of SNR for $n=$ $\{1,4,8,16,32\}$. Similar to the previous observation, the deterministic equivalent provides an accurate approximation for all values of SNR and $n$. In order to further verify our results, we have compared them against the expressions derived in [7, Theorem 1]. Both approximations, although not formally identical, turned out to yield almost identical results. However, we need to remark that there is no explicit algorithm provided in [7] to find a solution to the set of implicit equations. In several cases, the classical fixed-point algorithm did not converge to the correct result. Moreover, the result is not proved for non co-diagonalizable matrices $\mathbf{H}_{k} \mathbf{H}_{k}^{\mathrm{H}}$.

\section{CONCLUSION}

We have studied a class of wireless communication channels with random unitary signature/precoding matrices which can be used to model different types of CDMA and SDMA systems over frequency-selective and flat fading channels. We have provided deterministic approximations of the Stieltjes transform, the mutual information and the SINR at the output of the MMSE receiver, which are asymptotically accurate, almost surely. To compute these approximations, we have

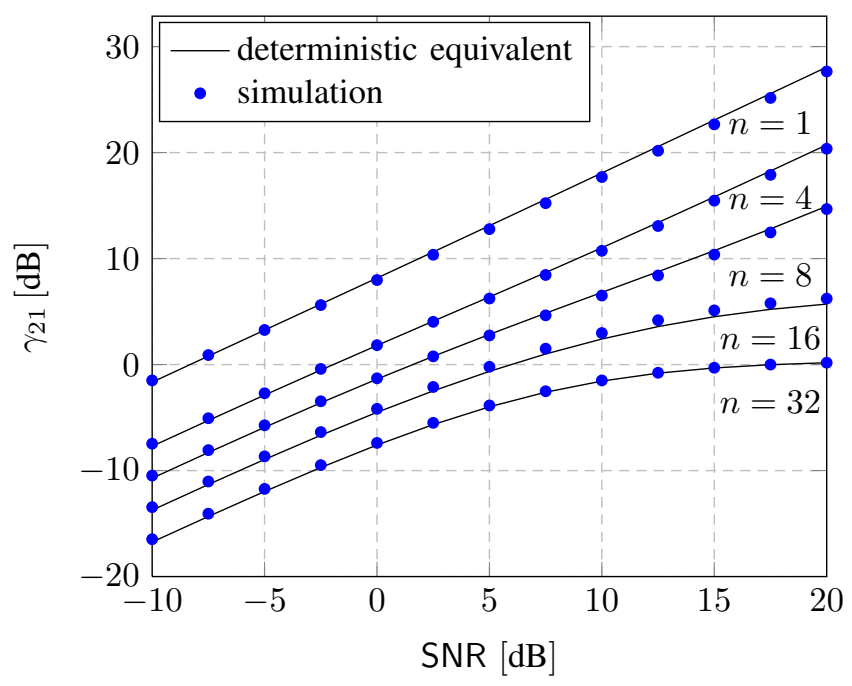

Fig. 3: SINR $\gamma_{21}$ at the output of the MMSE receiver for for $N=32$ and different numbers of transmit signatures $n$.

derived a simple fixed-point algorithm and proved its convergence to the correct solution. Our simulations verify the accuracy of the approximations for systems of even small dimensions. Since our analysis is not based on results from free probability theory and we do not require any of the involved matrices to be asymptotically free, our work is also a novel contribution to the field of random matrix theory. We also believe that the derived expressions will find useful applications to the study of future SDMA systems which are foreseen to apply unitary precoding codebooks.

\section{APPENDIX}

Sketch of proof of Theorem 1 and 2: Due to space limitations, we only give a sketch of proof for the case $\lim \sup _{k} n_{k} / N<1$. The case $\lim \sup _{k} n_{k} / N=1$ is provided together with the full proof in [15].

We wish to prove that there exists a matrix $\mathbf{F}=$ $\sum_{k=1}^{K} \bar{f}_{k} \mathbf{R}_{k}$, such that for all nonnegative $\mathbf{A}$ with $\|\mathbf{A}\|<\infty$ and $z<0$,

$$
\frac{1}{N} \operatorname{tr} \mathbf{A}\left(\mathbf{B}-z \mathbf{I}_{N}\right)^{-1}-\frac{1}{N} \operatorname{tr} \mathbf{A}\left(\mathbf{F}-z \mathbf{I}_{N}\right)^{-1} \underset{N \rightarrow \infty}{\stackrel{\text { a.s. }}{\longrightarrow}} 0 .
$$

At the heart of the derivation is the following trace lemma for Haar distributed matrices:

Lemma 1 ([1]): Let $\mathbf{W}$ be $n<N$ columns of a $N \times N$ Haar matrix and suppose $\mathbf{w}$ is a column of $\mathbf{W}$. Let $\mathbf{C}_{N}$ be a $N \times N$ random matrix, being a function of all columns of $\mathbf{W}$ except $\mathbf{w}$, and assume $\sup _{N}\left\|\mathbf{C}_{N}\right\|<\infty$. Then,

$\mathbf{w}^{\mathrm{H}} \mathbf{C}_{N} \mathbf{w}-\frac{1}{N-n} \operatorname{tr}\left(\mathbf{I}_{N}-\mathbf{W} \mathbf{W}^{\mathrm{H}}+\mathbf{w w}^{\mathrm{H}}\right) \mathbf{C}_{N} \underset{N \rightarrow \infty}{\stackrel{\text { a.s. }}{\longrightarrow}} 0$.

Contrary to classical deterministic equivalent approaches for random matrices with i.i.d. entries, finding a deterministic equivalent for $\frac{1}{N} \operatorname{tr} \mathbf{A}\left(\mathbf{B}-z \mathbf{I}_{N}\right)^{-1}$ is not straightforward. This is because terms of the form $\frac{1}{N-n_{k}} \operatorname{tr}\left(\mathbf{I}-\mathbf{W}_{k} \mathbf{W}_{k}^{\mathrm{H}}\right) \mathbf{A}^{1 / 2}(\mathbf{B}-z \mathbf{I})^{-1} \mathbf{A}^{1 / 2}$ will naturally 
appear in the derivation (as a consequence of Lemma 1) and need to be controlled. We proceed therefore as follows:

(i) Defining the random variables $(1 \leq k \leq K)$

$$
\begin{aligned}
\delta_{k} & =\frac{1}{N-n_{k}} \operatorname{tr}\left(\mathbf{I}_{N}-\mathbf{W}_{k} \mathbf{W}_{k}^{\mathrm{H}}\right) \mathbf{H}_{k}^{\mathrm{H}}\left(\mathbf{B}-z \mathbf{I}_{N}\right)^{-1} \mathbf{H}_{k} \\
f_{k} & =\frac{1}{N} \operatorname{tr} \mathbf{R}_{k}\left(\mathbf{B}-z \mathbf{I}_{N}\right)^{-1}
\end{aligned}
$$

and the matrix $\mathbf{G}=\sum_{k=1}^{K} \bar{g}_{k} \mathbf{R}_{k}$, we prove that

$$
f_{k}-\frac{1}{N} \operatorname{tr} \mathbf{R}_{k}\left(\sum_{l=1}^{K} \bar{g}_{l} \mathbf{R}_{l}-z \mathbf{I}_{N}\right)^{-1} \underset{N \rightarrow \infty}{\stackrel{\text { a.s. }}{\longrightarrow}} 0
$$

where $\bar{g}_{k}=\frac{1}{1-\frac{1}{N} \sum_{l=1}^{n_{k}} \frac{p_{l k} \delta_{k}}{1+p_{l k} \delta_{k}}} \frac{1}{N} \sum_{l=1}^{n_{k}} \frac{p_{l k}}{1+p_{l k} \delta_{k}}$.

(ii) Since the expression of $\bar{g}_{k}$ is not convenient to handle, we show as a next step that

$$
\bar{g}_{k}-\frac{1}{N} \operatorname{tr} \mathbf{P}_{k}\left(f_{k} \mathbf{P}_{k}+\left[1-f_{k} \bar{g}_{k}\right] \mathbf{I}_{n_{k}}\right)^{-1} \underset{N \rightarrow \infty}{\stackrel{\text { a.s. }}{\longrightarrow}} 0 \text {. }
$$

(iii) The relations (6) and (7) may be already sufficient to infer the deterministic equivalent, but can be made more attractive for further considerations. We therefore introduce the matrix $\mathbf{F}=\sum_{k=1}^{K} \bar{f}_{k} \mathbf{R}_{k}$ and prove that

$$
\begin{aligned}
& f_{k}-\frac{1}{N} \operatorname{tr} \mathbf{R}_{k}\left(\sum_{l=1}^{K} \bar{f}_{l} \mathbf{R}_{l}-z \mathbf{I}_{N}\right)^{-1} \underset{N \rightarrow \infty}{\stackrel{\text { a.s. }}{\longrightarrow} 0} \\
& \bar{f}_{k}-\frac{1}{N} \operatorname{tr} \mathbf{P}_{k}\left(f_{k} \mathbf{P}_{k}+\left[1-f_{k} \bar{f}_{k}\right] \mathbf{I}_{n_{k}}\right)^{-1}=0
\end{aligned}
$$

where $\bar{f}_{k}=\lim _{t \rightarrow \infty} x^{(t)}$ and $x^{(t)}$ is given by the fixedpoint algorithm

$$
x^{(t)}=\frac{1}{N} \operatorname{tr} \mathbf{P}_{k}\left(f_{k} \mathbf{P}_{k}+\left[1-f_{k} x^{(t-1)}\right] \mathbf{I}_{n_{k}}\right)^{-1}
$$

with $x^{(0)} \in\left[0, c_{k} / f_{k}\right)$. This means that $\bar{f}_{k}$ is uniquely determined by $f_{k}$. One can also verify that $x^{(t)} \in\left[0, c_{k} / f_{k}\right)$ for all $t$, and, thus, also $\bar{f}_{k} \in\left[0, c_{k} / f_{k}\right)$.

(iv) We then prove the existence and uniqueness of a solution to the following set of fixed-point equations:

$$
\begin{aligned}
& e_{k}=\frac{1}{N} \operatorname{tr} \mathbf{R}_{k}\left(\sum_{l=1}^{K} \bar{e}_{l} \mathbf{R}_{l}-z \mathbf{I}_{N}\right)^{-1} \\
& \bar{e}_{k}=\frac{1}{N} \operatorname{tr} \mathbf{P}_{k}\left(\bar{e}_{k} \mathbf{P}_{k}+\left[1-e_{k} \bar{e}_{k}\right] \mathbf{I}_{n_{k}}\right)^{-1}
\end{aligned}
$$

for all finite $N, z<0$ and $\bar{e}_{k} \in\left[0, c_{k} / e_{k}\right)$. While the existence of a solution follows from standard arguments (e.g. [14, Appendix A, Sec. C]), the uniqueness unfolds from a property of so-called standard functions [16]. More precisely, we show that the vector-valued function $\mathbf{h}=\left(h_{1}, \ldots, h_{K}\right)$ with $h_{k}:\left(x_{1}, \ldots, x_{K}\right) \mapsto$ $\frac{1}{N} \operatorname{tr} \mathbf{R}_{k}\left(\sum_{l=1}^{K} \bar{x}_{l} \mathbf{R}_{l}-z \mathbf{I}_{N}\right)^{-1}$ and $\bar{x}_{k}$ being the unique solution to

$$
\bar{x}_{k}=\frac{1}{N} \operatorname{tr} \mathbf{P}_{k}\left(\bar{x}_{k} \mathbf{P}_{k}+\left[1-x_{k} \bar{x}_{k}\right] \mathbf{I}_{n_{k}}\right)^{-1}
$$

lying in $\left[0, c_{k} / x_{k}\right)$, is a standard function. It follows then from [16, Lemma 1, Theorem 1] that the fixedpoint equation in $\left(e_{1}, \ldots, e_{K}\right)$ has a unique solution with positive entries and that this solution can be determined by iteration of the standard fixed-point Algorithm 1. This proves Theorem 1.

(v) The last step is to show that the unique solution $\left(e_{1}, \ldots, e_{N}\right)$ as provided by Theorem 1 satisfies

$$
e_{k}-f_{k} \underset{N \rightarrow \infty}{\stackrel{\text { a.s. }}{\longrightarrow}} 0 \text {. }
$$

This is done by standard arguments inspired by the proof of [13, Lemma 6.6]. Using the last result and the fact that $e_{k}-f_{k} \stackrel{\text { a.s. }}{\longrightarrow} 0$ implies $\bar{e}_{k}-\bar{f}_{k} \stackrel{\text { a.s. }}{\longrightarrow} 0$, it is straightforward to show (5). Choosing $\mathbf{A}=\mathbf{I}_{N}$ in (5) is sufficient to prove Theorem 2 (i). The proofs of Theorem 2 (ii) and (iii) do not require any novel arguments and are given in [15].

\section{REFERENCES}

[1] M. Debbah, W. Hachem, P. Loubaton, and M. de Courville, "MMSE Analysis of Certain Large Isometric Random Precoded Systems," IEEE Trans. Inf. Theory, vol. 49, no. 5, pp. 1293-1311, May 2003.

[2] J.-M. Chaufray, W. Hachem, and P. Loubaton, "Asymptotic Analysis of Optimum and Suboptimum CDMA Downlink MMSE Receivers," IEEE Trans. Inf. Theory, vol. 50, no. 11, pp. 2620-2638, Nov. 2004.

[3] M. Peacock, I. Collings, and M. Honig, "Asymptotic Analysis of LMMSE Multiuser Receivers for Multi-signature Multicarrier CDMA in Rayleigh Fading," IEEE Trans. Commun., vol. 52, no. 6, pp. 964 972, Jun. 2004.

[4] _ "Asymptotic Spectral Efficiency of Multiuser Multisignature CDMA in Frequency-selective Channels," IEEE Trans. Inf. Theory, vol. 52, no. 3, pp. 1113-1129, Mar. 2006.

[5] R. Couillet and M. Debbah, "Uplink Capacity of Self-organizing Clustered Orthogonal CDMA Networks in Flat Fading Channels," in Proc. Information Theory Workshop Fall'09 (ITW'09), Taormina, Sicily, 2009.

[6] _- Random Matrix Methods for Wireless Communications. Cambridge University Press, New York, to appear.

[7] M. Peacock, I. Collings, and M. Honig, "Eigenvalue Distributions of Sums and Products of Large Random Matrices Via Incremental Matrix Expansions," IEEE Trans. Inf. Theory, vol. 54, no. 5, pp. 2123-2138, May 2008.

[8] D. Love and R. Heath, "Limited Feedback Unitary Precoding for Spatial Multiplexing Systems," IEEE Trans. Inf. Theory, vol. 51, no. 8, pp. 2967-2976, Aug. 2005.

[9] J. Lee, J.-K. Han, and J. C. Zhang, "MIMO Technologies in 3GPP LTE and LTE-Advanced," EURASIP Journal on Wireless Communications and Networking, vol. 2009, May 2009.

[10] K. Huang, J. Andrews, and R. Heath, "Performance of Orthogonal Beamforming for SDMA With Limited Feedback," IEEE Trans. Veh. Technol., vol. 58, no. 1, pp. 152-164, Jan. 2009.

[11] T. Cover and J. A. Thomas, Elements of Information Theory, 2nd. Edition. John Wiley \& Sons, Inc., 2006.

[12] S. Verdú, Multiuser Detection. Cambridge University Press, New York, 1998.

[13] W. Hachem, P. Loubaton, and J. Najim, "Deterministic Equivalents for Certain Functionals of Large Random Matrices," Annals of Applied Probability, vol. 17, no. 3, pp. 875-930, 2007.

[14] R. Couillet, M. Debbah, and J. Silverstein, "A Deterministic Equivalent for the Analysis of Correlated MIMO Multiple Access Channels," IEEE Trans. Inf. Theory, 2010, accepted for publication. [Online]. Available: http://arxiv.org/abs/0906.3667

[15] R. Couillet, J. Hoydis, and M. Debbah, "A Deterministic Equivalent Approach to the Performance Analysis of Isometric Random Precoded Systems," IEEE Trans. Inf. Theory, Nov. 2010, submitted. [Online]. Available: http://arxiv.org/abs/1011.3717

[16] R. Yates, "A Framework for Uplink Power Control in Cellular Radio Systems," vol. 13, no. 7, pp. 1341-1347, Sep. 1995. 